\title{
Association between clinical symptoms and apolipoprotein A1 or apolipoprotein B levels is regulated by apolipoprotein $E$ variant rs429358 in patients with chronic schizophrenia
}

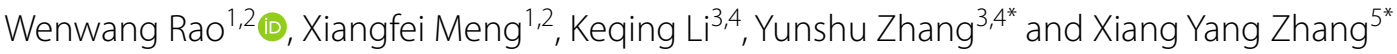

\begin{abstract}
Background: The apolipoprotein E (ApOE) gene polymorphisms are correlated with blood lipid levels and several neuropsychiatric symptoms. Therefore, this study aimed to examine whether the ApoE rs429358 affected the development and clinical symptoms of schizophrenia and to explore the relationship between apolipoproteins levels and clinical symptoms.

Methods: The ApoE rs429358 was genotyped using a case-control design. The Positive and Negative Syndrome Scale (PANSS) was employed to evaluate the psychopathology of all patients.

Results: A total of 637 patients with schizophrenia and 467 healthy controls were recruited. We found no significant differences in the genotype and allele distribution between the patient and control groups. A significant correlation between PANSS negative symptoms and ApoA1 levels $(p=0.048)$ or ApoB levels $(p=0.001)$ was found in patients with schizophrenia, which was also confirmed by linear regression analyses ( $p=0.048$ vs. $p=0.001$ ). Interestingly, only in the Thomozygote group, ApoA 1 and ApoB levels were predictors of the PANSS negative symptom score $(p=0.008$ vs. $p=0.012$ ), while in the $C$ allele carrier group, no correlation was observed.

Conclusions: This study found that the levels of $A p o A 1$ and $A p o B$ were negatively associated with negative symptoms of patients with schizophrenia. Furthermore, the association between ApoA1 or ApoB levels and psychopathology of schizophrenia was regulated by ApoE rs429358.
\end{abstract}

Keywords: Apolipoprotein A1, Apolipoprotein B, Apolipoprotein E, Clinical symptoms, Schizophrenia

\section{Introduction}

Schizophrenia is a severe long-term mental disease, with an estimated lifetime prevalence of $0.4 \% \sim 0.88 \%$ [1-4], and may lead to high disability [5] and potential years of life loss [6]. A systematic review showed that the economic burden of schizophrenia was between 0.02 and

\footnotetext{
*Correspondence: yunshucoffee@sina.com; zhangxy@psych.ac.cn ${ }^{3}$ Institute of Mental Health, Hebei Mental Health Centre, Hebei, China ${ }^{5}$ CAS Key Laboratory of Mental Health, Institute of Psychology, Chinese Academy of Sciences, 16 Lin Cui Road, Beijing 100101, China

Full list of author information is available at the end of the article
}

$1.65 \%$ (that is, US\$94 million-US\$102 billion) of the gross domestic product (GDP) [7]. The Global burden of Disease 2016 (GBD 2016) study estimated that the burden of schizophrenia accounts for $1.7 \%$ of total years of life lived with disability (YLD) globally [8]. Studies have shown that there is a close relationship between changes in serum or membrane lipids and pathophysiology [9]. Patients with schizophrenia are characterized by psychiatric symptoms [10], and changes in blood lipid profiles $[11,12]$. However, the biological mechanisms of original author(s) and the source, provide a link to the Creative Commons licence, and indicate if changes were made. The images or other third party material in this article are included in the article's Creative Commons licence, unless indicated otherwise in a credit line to the material. If material is not included in the article's Creative Commons licence and your intended use is not permitted by statutory regulation or exceeds the permitted use, you will need to obtain permission directly from the copyright holder. To view a copy of this licence, visit http://creativecommons.org/licenses/by/4.0/. The Creative Commons Public Domain Dedication waiver (http://creativeco mmons.org/publicdomain/zero/1.0/) applies to the data made available in this article, unless otherwise stated in a credit line to the data. 
underlying psychopathological symptoms and blood lipid profile changes in patients with schizophrenia remain unclear.

The Apolipoprotein E $(A p o E)$ gene (4 exons and 3 introns) is located at $19 \mathrm{q} 13.32$, and has been described as a major candidate gene involved in neuropsychiatric genetics $[13,14]$. It is well known that $A p o E$ gene plays a key role in receptor-mediated endocytosis of lipoproteins in the brain [15] and affects downstream proteins, such as brain-derived neurotrophic factor (BDNF) $[13,16]$. There are two functional polymorphism loci (rs429358 and rs7412) on exon 4 of $A p o E$ gene, which combine to form three main isoforms ( $\varepsilon 2, \varepsilon 3$, and $\varepsilon 4$; Fig. 1) [17]. The minor allele (i.e., C) frequency of $A p o E$ rs429358 from the Allele Frequency Aggregator (ALFA) project ranged from 0.026 in the East Asian population to 0.141 in the other African population [18]. The highest haplotype frequencies (rs440446- rs769449-rs769450-rs429358rs7412) of $A p o E$ gene in the 1000 Genomes were 0.292 (GGGCC) in the African population, 0.628 (CGGTC) in the Asian population, and 0.423 (GGATC) in the Europe population [19]. A previous systematic review and metaanalysis revealed the association between the ApoE \&3 and schizophrenia in Asian population [20]. In addition, there is evidence that ApoE $\varepsilon 4$ is associated with a variety of neuropsychiatric symptoms (e.g., depression, anxiety, apathy, agitation, aggression, hallucinations, and delusions) in patient with Alzheimer's disease [21-23]. Moreover, ApoE polymorphism may significantly affect lipid and lipoprotein levels [24]. In particular, one study found that ApoE rs429358 and ApoE rs7412 polymorphisms were significantly associated with low-density lipoproteins (LDL) levels in White and African Americans [25].

Clinical and epidemiological studies have found that changes in lipid profiles are also associated with a high risk of cardiovascular disease [26, 27], and schizophrenia is significantly associated with increased risk of cardiovascular diseases [28]. A previous study reported

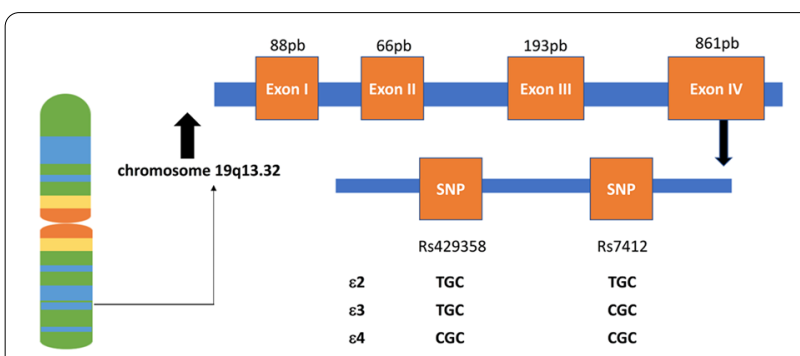

Fig. 1 Schematic diagram of the human ApoE gene. The ApoE gene is located on chromosome 19 with four exons and two non-synonymous SNPs (rs429358 and rs7412) in exon 4 generate three major allelic variants $(\varepsilon 2, \varepsilon 3$, and $\varepsilon 4)$ that changes in the blood lipid profiles were observed in patients with schizophrenia [29]. Apolipoprotein A1 (ApoA1) and apolipoprotein B (ApoB) are important components of apolipoproteins (Apos) and play a key role in lipid transport [30]. ApoA1 is the main protein component of high-density lipoproteins (HDL), and a biomarker of cardiovascular disease [31], while ApoB is a main protein in plasma chylomicrons, very-lowdensity lipoproteins (VLDL), and LDL [32]. The reference values of ApoA1 and ApoB levels in the adults ( $>18$ years) were $\geq 120 \mathrm{mg} / \mathrm{dL}$ and $\geq 100 \mathrm{mg} / \mathrm{dL}$ in males, and $\geq 140 \mathrm{mg} / \mathrm{dL}$ and $\geq 100 \mathrm{mg} / \mathrm{dL}$ in females [33]. The median ApoA1 and ApoB levels of health controls were $1.19 \mathrm{~g} / \mathrm{L}$ and $0.86 \mathrm{~g} / \mathrm{L}$ in the Africans, $1.15 \mathrm{~g} / \mathrm{L}$ and $0.98 \mathrm{~g} / \mathrm{L}$ in the Arabs, $1.23 \mathrm{~g} / \mathrm{L}$ and $0.83 \mathrm{~g} / \mathrm{L}$ in the Chinese, $1.26 \mathrm{~g} / \mathrm{L}$ and $0.95 \mathrm{~g} / \mathrm{L}$ in the Europeans, $1.18 \mathrm{~g} / \mathrm{L}$ and $0.95 \mathrm{~g} / \mathrm{L}$ in the Latin Americans, $1.07 \mathrm{~g} / \mathrm{L}$ and $0.92 \mathrm{~g} / \mathrm{L}$ in the South Asians, and $1.29 \mathrm{~g} / \mathrm{L}$ and $0.96 \mathrm{~g} / \mathrm{L}$ in the Southeast Asians, respectively [34]. A large number of studies have observed changes in the levels of ApoA1 and ApoB in patients with schizophrenia, with mixed results, such as elevated ApoA1 [31, 35, 36] or ApoB [37-39], which can be used as biomarkers of schizophrenia [39] as well as decreased ApoA1 [38, 40] or ApoB [36]. In addition, $A p o E$ gene polymorphism may affect the expression levels of ApoA1 and ApoB [41-43]. For example, one study found that $A p o E \& 4$ allele carriers had significantly higher levels of $A p o B$ in the community cohort of older individuals [42], while another study found that $A p o E \varepsilon 2$ allele carriers had significantly lower levels of ApoB in Chinese Han population [41]. Interestingly, the Alzheimer disease (AD) subjects carrying $A p o E$ \&4 allele had lower ApoA1 levels but higher ApoB levels [44] and there were differences in biomarker levels (e.g., cholesterol, LDL, and ApoB) for each $A p o E$ genotype with reference to $\varepsilon 3 \varepsilon 3$ in a UK Biobank study [45].

As mentioned above, we speculated that ApoE polymorphism rs429358 may be involved in the development of schizophrenia and further the relationship between the clinical symptoms and the ApoA1 or ApoB levels in patients with schizophrenia. Therefore, the main purposes of this study were (1) to identify the association between ApoE polymorphism rs429358 and schizophrenia; (2) to analyze the relationship between ApoA1 or ApoB levels and clinical symptoms in patients with schizophrenia; and (3) to explore whether the relationship between serum levels of ApoA1 or ApoB and clinical symptoms was regulated by $A p o E$ polymorphism rs429358. 


\section{Materials and methods Study participants}

Patients were recruited from Beijing Hui-Long-Guan hospital, and Hebei Rongjun Hospital in Baoding city near Beijing. They met the following inclusion criteria: (1) 18-70 years old; (2) diagnosed with schizophrenia by two psychiatrists using the Chinese version of Structured Clinical Interview for DSM-IV (SCID), with a Kappa value greater than 0.80 ; (3) having the diagnosis for at least 2 years to meet the eligibility of chronicity; (4) having taken a stable dose of oral antipsychotics for at least 12 months before entering this study; and (5) having no other psychiatric diagnoses.

In this study, healthy controls were randomly recruited through advertisements in the local community. They met the following inclusion criteria: (1) 18-70 years old; (2) no self-reported personal or family history of any type of psychiatric disorder; and (3) having not taken psychoactive drugs (e.g., antipsychotic, antianxiety, antidepressant, or mood-stabilizing drugs).

Participants were excluded if they met the following criteria: (1) diagnosed with drug or alcohol abuse/ dependence; (2) suffering from major physical diseases, including head injury, epilepsy, cardiovascular disease, cerebrovascular disease, infection, cancer, and diabetes; and (3) pregnancy.

All participants voluntarily participated in this study and gave written informed consent. The protocol was approved by the Ethics Committee of Beijing Hui-LongGuan hospital, and it was implemented in accordance with the Declaration of Helsinki [46].

\section{Data collection and measures}

General information, socio-demographic characteristics, and medical conditions were collected by trained researchers from self-designed questionnaires and medical records. Four psychiatrists used the Positive and Negative Syndrome Scale (PANSS) to assess the clinical symptoms of patients $[47,48]$ on the same day as the whole blood sampling. These psychiatrists received a training in the use of PANSS before the start of this study. After training, they maintained an inter-rater correlation coefficient (ICC) greater than 0.8 .

\section{DNA extraction and SNP genotyping}

Genomic DNA was extracted from $5 \mathrm{ml}$ of peripheral venous blood sample using the standard salting-out procedures [49], and then stored at -80 degrees. According to the protocol [50], ApoE polymorphism rs429358 was genotyped by using Matrix-Assisted Laser Desorption/ Ionization Time of Flight Mass Spectrometry (MALDITOF-MS) (Sequenom Inc., San Diego, CA, USA). After querying the reference sequence of the NCBI GenBank database, primers and extension probes were generated. Re-genotyping was performed by trained researchers without knowing the clinical information in 5\% randomly selected samples for quality control, with an error rate of less than $0.1 \%$.

\section{Serum ApoA1 and ApoB levels measurement}

Blood sampling and measurement of serum apolipoprotein (ApoA1 and ApoB) levels were described in detail in our previous studies $[12,51,52]$, which were performed on the Olympus AU2700 analyzer using the immunoturbidimetric method (Beijing Leadman Biotechnology).

\section{Statistical analysis}

The differences between cases and controls were measured by using independent two-sample t-test for continuous variables and Chi-squared $\left(\mathrm{x}^{2}\right)$ for categorical variables. The deviations from Hardy-Weinberg equilibrium (HWE) and genetic model (i.e., codominant, dominant, recessive, and over-dominant) analysis for ApoE rs429358 were examined using the SNPStats program, a web tool for SNP analysis (https://www.snpstats.net/ start.htm) [53]. Further, in order to identify the effect of ApoE rs429358 on susceptibility to schizophrenia, logistic regression was used to control for confounding factors.

Since almost no homozygous variant CC genotypes were detected in our study (approximately $0.8 \%$ of the patients and $0.5 \%$ of healthy controls), we considered the $\mathrm{CC}$ and TC genotypes as a group in the following association analysis.

Since all demographic and clinical variables, as well as ApoA1 and ApoB levels were normally distributed in patients and healthy controls (Kolmogorov-Smirnov one sample test, all $\mathrm{p}>0.05$ ). An independent two-sample $\mathrm{t}$-test was used to assess between-group differences grouped by genotypes for continuous variables, and Chisquared $\left(\chi^{2}\right)$ was used for dichotomous variables. Taking the ApoE rs429358 genotype as the independent variable and the PANSS total score and its subscale scores as the dependent variables, the analysis of covariance (ANCOVA) was further constructed, with those variables that showed significant differences in the above independent two-sample t-test or Chi-square test as covariates. Pearson correlation analysis was used to analyze the correlation between variables, and the partial correlation analysis was carried out with age, gender, education level, BMI, and age of onset as covariates. Linear regression analysis was used to explore whether there were differences in the relationships between ApoA1 or ApoB levels and PANSS scores among the ApoE rs429358 subgroups. The PANSS total or subscale scores were taken as 
dependent variables, and levels of $A p o A 1$ and $A p o B$ were used as independent variables for the whole sample and each $A p o E$ rs429358 subgroup, adjusting for age, gender, education, BMI, and age of onset. Bonferroni corrections were applied to each test to adjust for multiple testing.

Power analysis was conducted using software Quanto (Version 1.2.3) under log additive, recessive, and dominant models, assuming that the prevalence rate of schizophrenia in the population was $1 \%$. All data analyses were performed using SPSS, version 26.0 (IBM SPSS, IBM Corp., Armonk, NY, USA), with a significance level of 0.05 (two sided).

\section{Results}

\section{Sample characteristics}

A total of 637 patients with schizophrenia and 467 healthy controls were included. Their demographic and clinical information are summarized in Table 1. Most patients received monotherapy with antipsychotic drugs, including clozapine $(n=245)$, risperidone $(n=116)$, chlorpromazine $(n=32)$, perphenazine $(n=26)$, sulpiride $(n=20)$, quetiapine $(n=19)$, aripiprazole $(n=14)$, olanzapine $(n=7)$, and haloperidol $(n=7)$. The average dose of antipsychotics (equivalent to chlorpromazine) was $391.72 \pm 181.22 \mathrm{mg} /$ day [5456]. There were significant differences in gender, BMI, and age between patients and healthy controls (all $\mathrm{p}<0.05$ ), which were adjusted as covariates in the following analyses. In addition, there were sex differences in ApoA1 and ApoB levels $(t=-12.468, p<0.001$ vs. $t=-7.253, p<0.001$, Fig. 2) and no significant difference was found in ApoA1 and ApoB levels between typical antipsychotics and atypical antipsychotics $(t=-0.592, p=0.554$ vs. $t=1.181, p=0.238)$. Besides, sex differences were found in PANSS positive symptoms $(t=-4.322, p<0.001)$, PANSS negative symptoms $(t=5.598, P<0.001)$, and PANSS general psychopathology $(t=-3.670, p<0.001)$, except for PANSS total score $(t=-0.145, p=0.885)$.

\section{Association analysis of ApoE rs429358 with schizophrenia}

The allele frequencies and genetic models are presented in Table 2. The HWE test revealed that the genotype distributions of the ApoE rs429358 in both schizophrenia and controls were consistent with HWE (case: $p=0.52$; control: $p=1.0$; all: $p=0.82$ ). Both $A p o E$ rs429358 allele and genotype distributions between patients and healthy controls did not differ (both $p>0.05)$.

\section{Relationship between the ApoE rs429358 and clinical variables in patients}

Table 3 shows the demographic data, clinical variables, and PANSS scores in the ApoE rs429358 genotype subgroups. There was a difference in BMI between the ApoE rs429358 genotype subgroups $(p=0.034)$. In addition, after controlling for the BMI, there was a trend toward significant difference in PANSS positive

Table 1 Demographic profiles in patients with schizophrenia cases and controls (Mean \pm SD)

\begin{tabular}{|c|c|c|c|c|}
\hline Variables & $\begin{array}{l}\text { Cases } \\
(n=637)\end{array}$ & $\begin{array}{l}\text { Controls } \\
(n=467)\end{array}$ & $t / X^{2}$ & $p$ value \\
\hline Age (years) & $47.52 \pm 10.61$ & $44.94 \pm 13.63$ & -3.392 & 0.001 \\
\hline \multicolumn{5}{|l|}{ Sex } \\
\hline Female (\%) & $156(24.5)$ & $274(58.7)$ & 132.406 & $<0.001$ \\
\hline Male (\%) & $481(75.5)$ & $193(41.3)$ & & \\
\hline Years of education & $9.30 \pm 6.81$ & $9.66 \pm 5.33$ & 0.955 & 0.340 \\
\hline Body mass index & $24.45 \pm 4.00$ & $25.14 \pm 4.18$ & 2.538 & 0.011 \\
\hline Age of onset (years) & $23.20 \pm 5.16$ & - & - & - \\
\hline Duration of illness (years) & $24.43 \pm 10.52$ & - & - & - \\
\hline $\begin{array}{l}\text { Mean daily dose (mg/day) } \\
\text { (chlorpromazine equivalents) }\end{array}$ & $391.72 \pm 181.22$ & - & - & - \\
\hline PANSS total score & $60.81 \pm 14.91$ & - & - & - \\
\hline Positive symptoms & $11.90 \pm 5.15$ & - & - & - \\
\hline Negative symptoms & $23.25 \pm 8.50$ & - & - & - \\
\hline General psychopathology & $25.66 \pm 6.02$ & - & - & - \\
\hline ApoA1 level (g/L) & $1.53 \pm 0.38$ & - & - & - \\
\hline ApoB level (g/L) & $0.89 \pm 0.24$ & - & - & - \\
\hline ApoA1/ApoB & $1.82 \pm 0.62$ & - & - & - \\
\hline
\end{tabular}




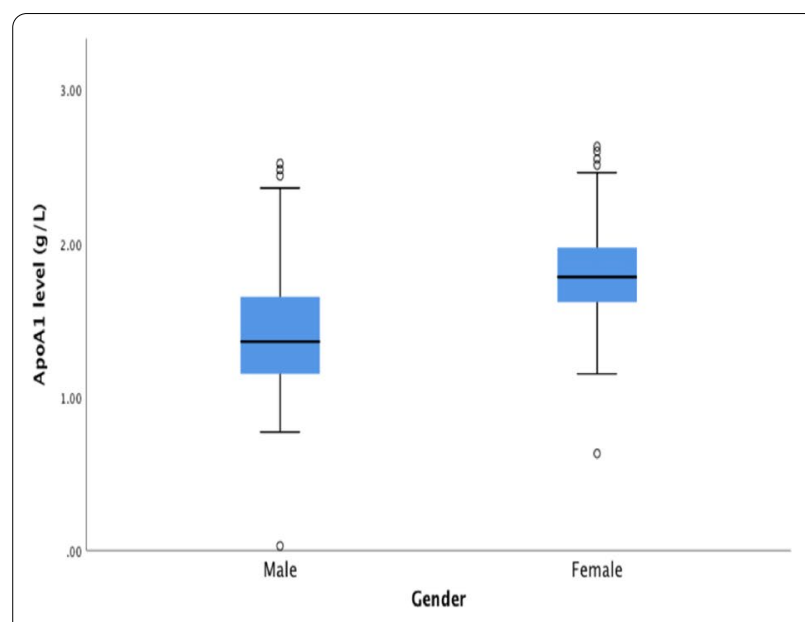

(a) ApoA1 levels

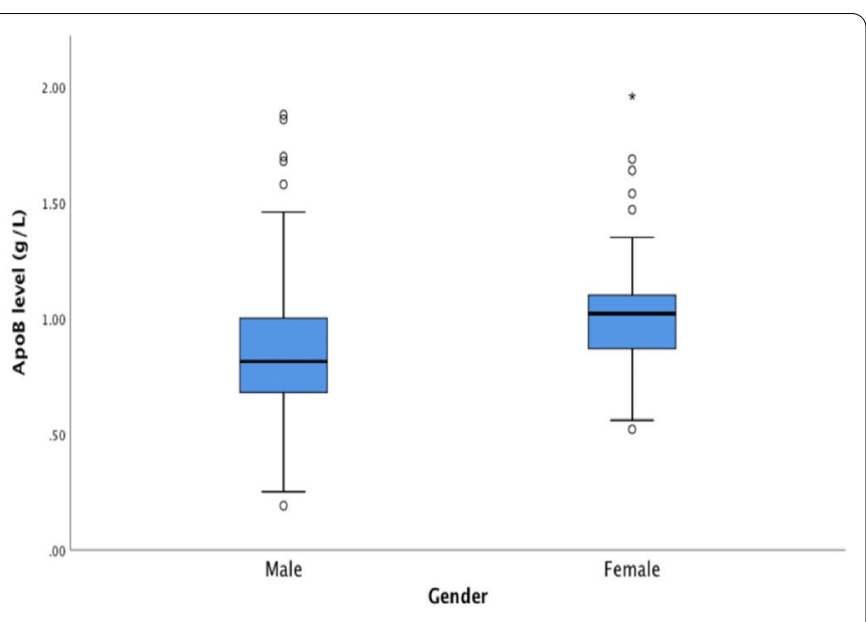

(b) ApoB levels

Fig. 2 The $A p o A 1(g / / L)$ and $A p o B$ levels $(g \mid / L)$ between the males and females

Table 2 Comparison of rs429358 allele and genotype frequencies between schizophrenia cases and controls

\begin{tabular}{|c|c|c|c|c|c|c|c|}
\hline Models & Genotypic & Cases & Controls & $x^{2}$ & OR $(95 \% \mathrm{Cl})$ & $p$ vaule & $p$ adjusted* \\
\hline \multicolumn{8}{|l|}{ Allele } \\
\hline & C (n\%) & $77(8.0)$ & $69(8.0)$ & 0.218 & $0.92(0.66-1.29)$ & 0.64 & 0.71 \\
\hline & $T(n \%)$ & $935(92.0)$ & 773 (92.0) & & 1.00 & & \\
\hline \multicolumn{8}{|c|}{ Codominant } \\
\hline & CC (n\%) & $4(0.8)$ & $2(0.5)$ & 0.922 & $1.64(0.30-8.98)$ & 0.63 & 0.12 \\
\hline & CT (n\%) & $69(13.6)$ & 65 (15.4) & & $0.87(0.60-1.25)$ & & \\
\hline & TT (n\%) & $433(85.6)$ & $354(84.1)$ & & 1.00 & & \\
\hline \multicolumn{8}{|c|}{ Dominant } \\
\hline & TT (n\%) & $433(85.6)$ & $354(84.1)$ & 0.396 & 1.00 & 0.53 & 0.45 \\
\hline & $C C+C T(n \%)$ & $73(14.4)$ & 67 (15.9) & & $0.89(0.62-1.28)$ & & \\
\hline \multicolumn{8}{|c|}{ Recessive } \\
\hline & $\mathrm{TT}+\mathrm{CT}(\mathrm{n} \%)$ & $502(99.2)$ & $419(99.5)$ & 0.348 & 1.00 & 0.55 & 0.08 \\
\hline & CC (n\%) & $4(0.8)$ & $2(0.5)$ & & $1.67(0.30-9.16)$ & & \\
\hline \multicolumn{8}{|c|}{ Over-dominant } \\
\hline & $\mathrm{TT}+\mathrm{CC}(\mathrm{n} \%)$ & $437(86.4)$ & $356(84.6)$ & 0.603 & 1.00 & 0.44 & 0.25 \\
\hline & CT (n\%) & 69 (13.6) & 65 (15.4) & & $\begin{array}{l}0.87 \\
(0.60-1.25)\end{array}$ & & \\
\hline
\end{tabular}

*Adjusted by sex, age, and BMI

symptom score $(p=0.073)$, PANSS negative symptom score $(p=0.097)$, and PANSS total score $(p=0.087)$ between the ApoE rs429358 genotype subgroups.

\section{Genotype effects on serum ApoA1 and ApoB levels between patients}

ApoA1 levels were available for 507 patients, while ApoB levels were available for 505 patients. There were no ApoA1 and ApoB levels available for healthy controls. There was a significant difference in serum ApoA1 levels between the ApoE rs429358 genotype groups, showing that patients with TT genotype had higher ApoA1 levels than patients with $\mathrm{CT}+\mathrm{CC}$ genotype $(p=0.010)$. After adjusting for BMI, the difference was still significant $(p=0.005)$. In addition, there was no significant difference in ApoB levels between the ApoE rs429358 genotype groups $(p=0.545)$.

\section{The effect of ApoE rs429358 on the relationship} between ApoA1 levels and PANSS scores in patients Pearson's correlation analysis indicated that ApoA1 levels were positively correlated with PANSS positive symptom 
Table 3 Demographic and clinical information according to ApoE rs429358 genotype of cases and controls in a Chinese sample

\begin{tabular}{|c|c|c|c|c|c|c|c|c|c|}
\hline \multirow[t]{2}{*}{ Variables } & \multicolumn{5}{|l|}{ Cases $(n=506)$} & \multicolumn{4}{|c|}{ Health Controls $(n=421)$} \\
\hline & $\begin{array}{l}\mathrm{CT}+\mathrm{CC} \\
(\mathrm{n}=73)\end{array}$ & $\begin{array}{l}\text { TT } \\
(n=433)\end{array}$ & $t / X^{2}$ & $p$ value & $p$ adjusted ${ }^{*}$ & $\begin{array}{l}\mathrm{CT}+\mathrm{CC} \\
(\mathrm{n}=67)\end{array}$ & $\begin{array}{l}\text { TT } \\
(\mathrm{n}=354)\end{array}$ & $t / X^{2}$ & $\begin{array}{l}p \\
\text { value }\end{array}$ \\
\hline Age (years) & $47.45 \pm 10.04$ & $47.36 \pm 9.66$ & -0.077 & 0.939 & - & $46.15 \pm 13.14$ & $46.16 \pm 13.27$ & 0.006 & 0.995 \\
\hline \multicolumn{10}{|l|}{ Sex } \\
\hline Female (\%) & $19(26.0)$ & $91(21.0)$ & 0.922 & 0.337 & - & $38(56.7)$ & $210(59.3)$ & 0.158 & 0.691 \\
\hline Male (\%) & $54(74.0)$ & $342(79.0)$ & & & & $29(43.3)$ & $144(40.7)$ & & \\
\hline Years of eudcation & $10.21 \pm 10.91$ & $8.72 \pm 2.60$ & -1.159 & 0.250 & - & $10.80 \pm 11.52$ & $9.14 \pm 3.32$ & -1.164 & 0.248 \\
\hline Body mass index & $23.55 \pm 4.12$ & $24.73 \pm 3.85$ & 2.131 & 0.034 & - & $25.00 \pm 3.58$ & $25.41 \pm 4.20$ & 0.734 & 0.464 \\
\hline Age of onset (years) & $23.34 \pm 5.58$ & $22.98 \pm 4.77$ & -0.587 & 0.558 & - & - & - & - & - \\
\hline Duration of illness (years) & $24.11 \pm 9.56$ & $24.53 \pm 10.07$ & 0.335 & 0.738 & - & - & - & - & - \\
\hline $\begin{array}{l}\text { Mean daily dose (mg/day) } \\
\text { (chlorpromazine equivalents) }\end{array}$ & $411.30 \pm 180.56$ & $386.20 \pm 167.21$ & -1.012 & 0.312 & - & - & - & - & - \\
\hline \multicolumn{10}{|l|}{ PANSS score } \\
\hline Positive symptoms & $12.34 \pm 5.19$ & $11.52 \pm 4.71$ & -1.357 & 0.175 & 0.073 & - & - & - & - \\
\hline Negative symptoms & $25.16 \pm 8.63$ & $23.45 \pm 8.36$ & -1.608 & 0.109 & 0.097 & - & - & - & - \\
\hline General psychopathology & $26.32 \pm 6.09$ & $25.47 \pm 5.92$ & -1.117 & 0.264 & 0.777 & - & - & - & - \\
\hline Total score & $63.82 \pm 14.51$ & $60.45 \pm 14.39$ & -1.849 & 0.065 & 0.087 & - & - & - & - \\
\hline ApoA1 level ( g/L) & $1.38 \pm 0.40$ & $1.52 \pm 0.37$ & 2.586 & 0.010 & - & - & - & - & - \\
\hline ApoB level ( $g / L)$ & $0.86 \pm 0.26$ & $0.88 \pm 0.26$ & 0.606 & 0.545 & - & - & - & - & - \\
\hline ApoA1/ApoB & $1.71 \pm 0.59$ & $1.84 \pm 0.64$ & 1.448 & 0.148 & - & - & - & - & - \\
\hline
\end{tabular}

* Adjusted by BMI

score $(r=0.142, n=494, p=0.001$, pcor $=0.004)$ and PANSS general psychopathology score $(r=0.152$, $n=494, p=0.001$, pcor $=0.004)$, while ApoA1 level was negatively correlated with the PANSS negative symptom score $(r=-0.160, n=494, p<0.001$, pcor $<0.004)$. After adjusting for age, sex, BMI, years of education, and age of onset, only a significant partial correlation was identified between the ApoA1 levels and the PANSS negative symptom score $(r=-0.110, n=323, p=0.048$, pcor $=0.192$, Fig. 3). Furthermore, linear regression analysis identified that ApoA1 level was a predictor of PANSS negative symptom score $(t=-1.988, p=0.048$, pcor $=0.192$ ).

Additionally, only in the $\mathrm{T}$ homozygote group, ApoA1 levels were positively correlated PANSS positive symptom score $(r=0.203, n=335, p<0.001$, pcor $<0.004)$

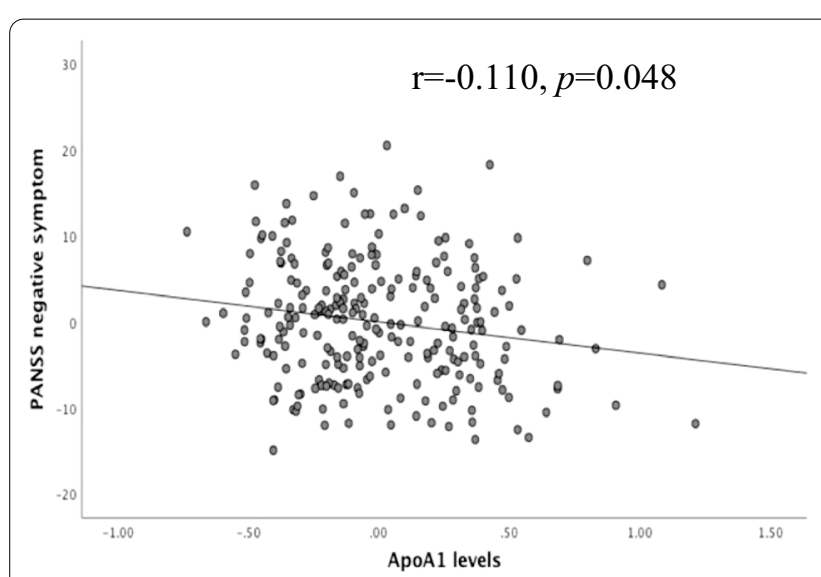

(a)

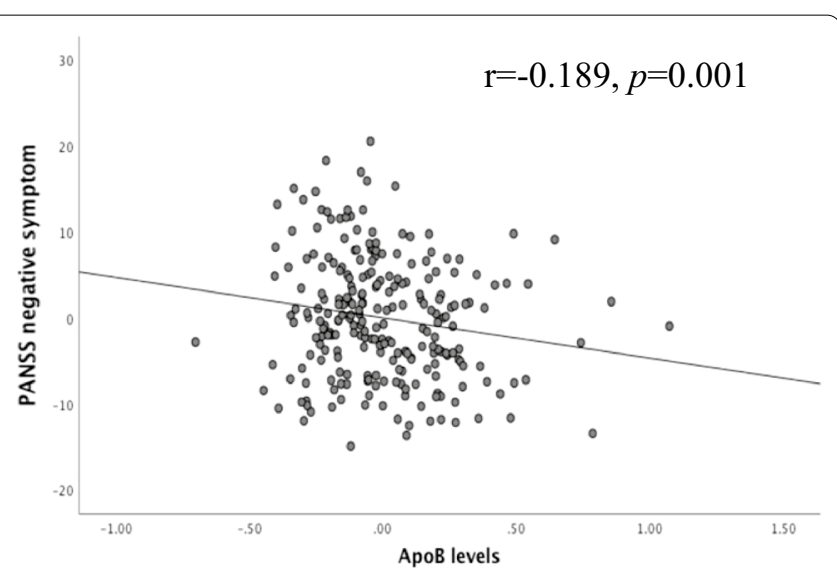

(b)

Fig. 3 The partial correlation between ApoA1 ( $\mathrm{g} / / \mathrm{L}$ ) and ApoB levels ( $\mathrm{g} / \mathrm{L}$ ) and PANSS scores of patients with schizophrenia. a ApoA1 levels and PANSS negative symptoms; $\mathbf{b}$ ApoB levels and PANSS negative symptoms 
and PANSS general psychopathology score $(r=0.219$, $n=335, p<0.001$, pcor $<0.004)$, but negatively correlated with the PANSS negative symptom score $(r=-0.165$, $n=335, p=0.002$, pcor $=0.008)$. Partial correlation analysis revealed that adjusting for age, gender, BMI, years of education, and age of onset, only the PANSS negative symptom score was correlated with the ApoA1 level $(r=-0.130, n=236, p=0.045$, pcor $=0.180)$. Moreover, linear regression analysis confirmed that ApoA1 level was a predictor of PANSS negative symptom score in patients with $\mathrm{T}$ homozygotes $(t=-2.694, p=0.008$, pcor $=0.032$ ).

Only in the $\mathrm{C}$ allele carriers, there was a trend toward significant correlation between PANSS negative symptom score and ApoA1 levels $(p=0.082$, pcor $=0.328)$. While controlling for age, gender, BMI, years of education, and age of onset, no correlation was observed between the PANSS negative symptom score and the ApoA1 levels $(p=0.127$, pcor $=0.508)$, which was also confirmed by linear regression analysis $(t=-1.562$, $p=0.127$, pcor $=0.508$ ).

\section{The effect of ApoE rs429358 on the relationship between ApoB levels and PANSS scores in patients}

Pearson's correlation showed that ApoB levels were negatively association with PANSS negative symptom score $(r=-0.231, n=492, p<0.001$, pcor $<0.004)$ or PANSS total score $(r=-0.105, n=492, p=0.020$, pcor $=0.080)$. After controlling for age, gender, BMI, years of education and age of onset, there was still a significant correlation between the PANSS negative symptom score and ApoB levels $(r=-0.189, n=321$, $p=0.001$, pcor $=0.004$, Fig. 3). Further linear regression analysis identified that ApoB level was a predictor of PANSS negative symptom score $(t=-3.447$, $p=0.001$, pcor $=0.004)$. In addition, only in the $\mathrm{T}$ homozygote group, a significant correlation was observed between the ApoB levels and PANSS negative symptom score in all patients $(r=-0.209, n=334$, $p<0.001$, pcor $<0.004)$. Further linear regression analyses confirmed that $A p o B$ level was a predictor of PANSS negative symptom score $(t=-2.529, p=0.012$, pcor $=0.048$ ).

Partial correlation analysis revealed that after adjusting for age, gender, BMI, years of education, and age of onset, only the PANSS negative symptom score was correlated with ApoB levels $(r=-0.1307, n=235$, $p=0.036$, pcor $=0.144$ ). Only in the $C$ allele carriers, there was a significant correlation between the PANSS negative symptom score and ApoB levels $(r=-0.274$, $n=57, \quad p=0.039$, pcor $=0.156)$ However, controlling for age, gender, BMI, years of education, and age of onset, no correlation was observed between the PANSS negative symptom score and ApoB levels $(p=0.072$, pcor $=0.288)$, which was also confirmed by linear regression analysis $(t=-1.856, p=0.072$, pcor $=0.288$ ).

\section{Discussion}

To our best knowledge, this is the first report to explore the relationship between ApoE rs429358 and clinical symptoms of patients with schizophrenia. We did not find an association between ApoE rs429358 and susceptibility to schizophrenia or clinical symptoms of schizophrenia. However, we found a significant association between serum ApoA1 or serum ApoB levels and PANSS negative symptoms, which was regulated by $A p o E$ rs429358.

\section{No association between ApoE rs429358 and susceptibility and clinical symptoms of schizophrenia}

This study revealed that there was no association between ApoE rs429358 and schizophrenia, which was similar to some previous studies $[57,58]$. However, a previous meta-analysis reported an association of ApoE $\varepsilon 3$ with schizophrenia in an Asian population [20]. Another study also found that there was a highly significant association between $A p o E$ genotype and schizophrenia in a Chinese population [59]. Moreover, a French association study and meta-analysis showed that only in a male sample, there was an association between schizophrenia and $A p o E$ \&2ع3 genotype [60]. In addition, an association between undifferentiated type of schizophrenia and $A p o E \varepsilon 3 / \varepsilon 3$ genotype was found in a Serbian population [61]. Both ApoE $\varepsilon 3$ and ApoE-219G haplotypes increased the risk of schizophrenia in siblings [62]. By comparing the above studies, we speculate that these different results between our current study and other studies may be partly due to differences in population, sample composition, type of schizophrenia, and synergy/interaction effects with other variants. Also, the age of patients could be a potential reason, and a meta-analysis indicated that $A p o E-\varepsilon 4$ may play an age-mediated pathophysiological role in schizophrenia [63].

In disagreement with previous studies, our study found no association between ApoE rs429358 and any PANSS scores. One previous study indicated that the phenotypes of drug-free schizophrenia patients with and without the $A p o E \varepsilon 4$ allele were different, showing that $A p o E$ $\varepsilon 4+$ patients had lower positive symptoms as measured by the Brief Psychiatric Rating Scale compared to ApoE ع4- patients [58]. Another study found that the frequency of the $\varepsilon 4$ allele was significantly higher in schizophrenia patients with positive symptoms [64]. A study also found that $A p o E \varepsilon 2$ genotype was associated with more 
depressive symptoms in older Chinese schizophrenia patients[65]. One possible reason for this is that the combined effect of rs429358 and rs7412, but not rs429358 alone, has a significant effect on the PANSS score of patients with schizophrenia. Furthermore, a longitudinal study found that $A p o E-\varepsilon 4$ predicted worsening severity of hallucinations and delusions in patients with schizophrenia in late adulthood [63]. Hence, it is worth to perform a follow-up study between ApoE rs429358 and subsequent PANSS scores.

\section{Serum ApoA1 levels, ApoB levels, and ApoE rs429358 genotype groups in patients}

Our finding that ApoA1 levels were significantly different between ApoE rs429358 subgroups. However, our study only showed that ApoB levels in TT genotype carriers were higher than those in $\mathrm{CT}+\mathrm{CC}$ carriers, but the differences were not significant between the ApoE rs429358 subgroups. These findings are not in agreement with a study that found significantly lower ApoA1 levels but higher ApoB levels in Alzheimer disease (AD) patients carrying ApoE \&4 allele [44]. Potential reasons included differences in disease (schizophrenia vs. AD) and different composition of variants (rs429358 alone vs. a combination of rs429358 and rs7412).

Numerous studies in the general population have found that ApoA1 levels and ApoB levels are different between ApoE rs429358 genotype subgroups. To demonstrate this, one study found that $A p o E \varepsilon 4$ allele carriers had significantly higher levels of ApoB in a community-based older individuals [42], while another study found that $A p o E \varepsilon 2$ allele carriers had significantly lower levels of ApoB in Chinese Han population [41]. Equally important, a UK Biobank study revealed that ApoA and ApoB levels were found to be significantly different for each $A p o E$ genotype with reference to $\varepsilon 3 \varepsilon 3$ [45]. The disease population (healthy persons vs. chronic schizophrenia patients) may be a major possible explanation. It is well known that some antipsychotic drugs (e.g., clozapine, olanzapine, etc.) may affect the body's metabolic levels $[66,67]$.

\section{Association between PANSS negative symptom score and ApoA 1 and ApoB levels}

Our study found that ApoA1 and ApoB levels were positively associated with PANSS negative symptoms in patients with schizophrenia. Similarly, a previous study showed that ApoA1 levels were negatively associated with psychopathology score (i.e., unusual thought content, grandiosity, elated mood, motor hyperactivity, and distractibility), while ApoB levels were positively associated with psychopathology (i.e., grandiosity, elated mood, motor hyperactivity) in male patients with psychosis [68]. In addition, there was a significant association between tardive dyskinesia (TD) status and ApoA1 and ApoB levels [69] as well as between cognitive function and ApoA1 and ApoB levels [70]. A possible hypothesis is that the improvement in psychopathology that accompanies changes in ApoA1 and ApoB levels may be a side effect indicating the efficacy of antipsychotic drugs, similar to the effect of antipsychotic-induced weight gain [51, 71]. A number of literatures have revealed that obesity increases the ApoB /ApoA1 ratio [72] and that ApoA1 levels were positively associated with the risk of metabolically healthy obesity (MHO), but the ApoB /ApoA1 ratio is negatively associated [73].

\section{Association between serum ApoA1 or ApoB levels and PANSS negative symptom score was regulated by ApoE rs429358}

Our study found that ApoE rs429358 genotype groupings had a significant effect on the relationship between serum ApoA1 or ApoB levels and negative symptoms of schizophrenia. The correlation between serum ApoA1 or ApoB levels and negative symptoms was observed only in the $\mathrm{T}$ homozygote group but not in the $\mathrm{C}$ allele group, which may provide a clue to the unusual findings that higher ApoA1 or ApoB levels were associated with better PANSS negative symptoms. There is evidence that some neuropsychiatric symptoms (e.g., depression, anxiety, apathy, agitation, aggression, hallucinations, and delusions) in $\mathrm{AD}$ patients are associated with the ApoE $\varepsilon 4$ allele [21-23]. Furthermore, in our study, given that TT genotype carriers had higher serum ApoA1 levels than CT + CC carriers, this indicates that the ApoE rs429358 polymorphism may regulate ApoA1 levels. Moreover, the regulatory effect of the rs 429358 polymorphism on ApoB levels may be subtle but still effectively affects the relationship between ApoB levels and psychiatric symptoms. Thus, ApoE genotype may play a role in conferring negative symptoms in schizophrenia by affecting ApoA1 or ApoB levels. We speculate that since negative symptoms are associated with certain brain structures (e.g., right frontal cortical surface area) [74] and ApoE genotype appears to be associated with whole brain structures [75], $A p o E$ genotype also may indirectly influence the above relationships by modulating brain structures. However, we still need to further explore the specific mechanisms by which ApoE rs429358 affects the association between ApoB levels and psychiatric symptoms.

This study had several limitations. First, owing to the cross-sectional study design, the causality between ApoE rs429358, ApoA1 and ApoB levels, clinical symptoms in patients with schizophrenia was not directly presented. Second, the inpatients in this study had more severe psychopathology, longer duration of disease, and antipsychotic treatment than typical psychiatric 
outpatients or first episode and drug-naive patients with schizophrenia, which may limit the generalization of our findings in this study. Third, we only examined a single genetic polymorphism effect in this study, and we need to detect other functional variants of the $A p o E$ gene, especially rs7412, because other polymorphisms, haplotypes, genes interaction, or gene-environment interaction may be associated with schizophrenia or with psychopathological symptoms of schizophrenia. Fourth, some potential covariates that may influence the blood lipid profile, such as dietary habits, physical exercise, and relevant treatments were not collected in the study. Fifth, we assessed serum apolipoprotein levels only in the case group, but not in the control group. Therefore, the observed relation between $A p o E$ variant status and alterations in ApoA1 levels is specific for patients with schizophrenia. Sixth, the relatedly small sample size in this study may impact the generalization of the findings.

\section{Conclusions}

In conclusion, ApoE rs429358 gene polymorphism did not directly affect the susceptibility to schizophrenia or psychiatric symptoms of schizophrenia. The association between serum ApoA1 and ApoB levels and clinical symptoms in patients with schizophrenia was regulated by the presence of ApoE rs429358 polymorphism. Nevertheless, due to the limited sample size and relatively low statistical power, our findings are still preliminary. Therefore, future studies are needed to confirm our current findings in larger samples from different ethnics, and the biological mechanisms of clinical symptoms of schizophrenia involved in ApoE rs429358 should be further studied.

\begin{abstract}
Abbreviations
ApoE: Apolipoprotein E; ApoA1: Apolipoprotein A1; ApoB: Apolipoprotein B; PANSS: Positive and Negative Syndrome Scale; GDP: Gross domestic product; GBD: Global burden of disease; YLD: Years of life lived with disability; BDNF: Brain-derived neurotrophic factor; ALFA: Allele Frequency Aggregator; LDL: Low-density lipoproteins; HDL: High-density lipoproteins; VLDL: Very-low-density lipoproteins; AD: Alzheimer disease; SCID: Structured Clinical Interview for DSM-IV; ICC: Inter-rater correlation coefficient; MALDI-TOF-MS: Matrix-Assisted Laser Desorption/lonization Time of Flight Mass Spectrometry; HWE: HardyWeinberg equilibrium; ANCOVA: Analysis of covariance; TD: Tardive dyskinesia; $\mathrm{MHO}$ : Metabolically Healthy Obese.
\end{abstract}

\section{Acknowledgements}

The authors would like to thank the mental health professionals and subjects who were involved in this study.

\section{Authors' contributions}

WWR and XYZ designed the study, WWR undertook the statistical analysis and wrote the first draft of the manuscript, and XFM and XYZ revised the manuscript. All the authors contributed to and have approved the final manuscript.

\section{Funding}

This work was funded by the National Natural Science Foundation of China (No.81371477), the Key R\&D Program of Science and Technology Department of Hebei Province (172777223), and Government-funded Subsidy Project for Specialty Capacity-Building and Specialist Leaders (361014).

\section{Data availability}

Data are available on reasonable request from the authors.

\section{Declarations}

Ethics approval

All procedures performed in these studies involving human participants were in accordance with the ethical standards of the Ethics Committee of Beijing Hui-Long-Guan hospital and adhere to the ethical standards laid down in the 1964 Declaration of Helsinki and its later amendments.

\section{Informed consent}

Informed consent forms were signed by participating individuals or their guardians.

\section{Consent for publication}

All the authors have consented to publication of this research.

\section{Competing interest}

There is no conflict of interest in the information contained in this manuscript.

\section{Author details}

${ }^{1}$ Department of Psychiatry, Faculty of Medicine and Health Sciences, McGill University, Montreal, QC, Canada. ${ }^{2}$ Mental Health and Society Division, Douglas Mental Health University Institute, Montreal, QC, Canada. ${ }^{3}$ Institute of Mental Health, Hebei Mental Health Centre, Hebei, China. ${ }^{4}$ Department of Sleep Medicine, Hebei Psychiatric Hospital, Hebei, China. ${ }^{5}$ CAS Key Laboratory of Mental Health, Institute of Psychology, Chinese Academy of Sciences, 16 Lin Cui Road, Beijing 100101, China.

Received: 20 September 2021 Accepted: 30 November 2021

Published online: 20 December 2021

\section{References}

1. Saha S, Chant D, Welham J, McGrath J. A systematic review of the prevalence of schizophrenia. PLoS Med. 2005;2(5):e141.

2. Moreno-Küstner B, Martín C, Pastor L. Prevalence of psychotic disorders and its association with methodological issues. A systematic review and meta-analyses. PLoS ONE. 2018;13(4):e0195687.

3. Phanthunane P, Vos T, Whiteford H, Bertram M, Udomratn P. Schizophrenia in Thailand: prevalence and burden of disease. Popul Health Metrics. 2010;8(1):24.

4. Huang Y, Wang Y, Wang H, Liu Z, Yu X, Yan J, Yu Y, Kou C, Xu X, Lu J. Prevalence of mental disorders in China: a cross-sectional epidemiological study. Lancet Psychiatry. 2019;6(3):211-24.

5. Vos T, Barber RM, Bell B, Bertozzi-Villa A, Biryukov S, Bolliger I, Charlson F, Davis A, Degenhardt L, Dicker D. Global, regional, and national incidence, prevalence, and years lived with disability for 301 acute and chronic diseases and injuries in 188 countries, 1990-2013: a systematic analysis for the Global Burden of Disease Study 2013. Lancet. 2015;386(9995):743-800.

6. Hjorthøj C, Stürup AE, MCGrath JJ, Nordentoft M. Years of potential life lost and life expectancy in schizophrenia: a systematic review and metaanalysis. Lancet Psychiatry. 2017:4(4):295-301.

7. Chong HY, Teoh SL, Wu DB-C, Kotirum S, Chiou CF, Chaiyakunapruk N. Global economic burden of schizophrenia: a systematic review. Neuropsychiatr Dis Treat. 2016;12:357.

8. Charlson FJ, Ferrari AJ, Santomauro DF, Diminic S, Stockings E, Scott JG, McGrath JJ, Whiteford HA. Global epidemiology and burden of schizophrenia: findings from the global burden of disease study 2016. Schizophr Bull. 2018;44(6):1195-203. 
9. Mesa-Herrera F, Taoro-González L, Valdés-Baizabal C, Diaz M, Marín R. Lipid and lipid raft alteration in aging and neurodegenerative diseases: a window for the development of new biomarkers. Int J Mol Sci. 2019;20(15):3810.

10. Rao W, Du X, Zhang Y, Yu Q, Hui L, Yu Y, Kou C, Yin G, Zhu X, Man L. Association between forkhead-box P2 gene polymorphism and clinical symptoms in chronic schizophrenia in a Chinese population. J Neural Transm. 2017;124(7):891-7.

11. Yogaratnam J, Biswas N, Vadivel R, Jacob R. Metabolic complications of schizophrenia and antipsychotic medications-an updated review. East Asian Arch Psychiatry. 2013;23(1):21.

12. An H-M, Tan Y-L, Tan S-P, Shi J, Wang Z-R, Yang F-D, Huang X-F, Soars JC, Kosten TR, Zhang X-Y. Smoking and serum lipid profiles in schizophrenia. Neurosci Bull. 2016;32(4):383-8.

13. Forero DA, López-León $S$, González-Giraldo Y, Dries DR, Pereira-Morales AJ, Jiménez KM, Franco-Restrepo JE. APOE gene and neuropsychiatric disorders and endophenotypes: a comprehensive review. Am J Med Genet B Neuropsychiatr Genet. 2018;177(2):126-42.

14. Villeneuve S, Brisson D, Marchant NL, Gaudet D. The potential applications of Apolipoprotein E in personalized medicine. Front Aging Neurosci. 2014;6:154

15. Puglielli L, Tanzi RE, Kovacs DM. Alzheimer's disease: the cholesterol connection. Nat Neurosci. 2003;6(4):345-51.

16. Holtzman DM, Herz J, Bu G. Apolipoprotein E and apolipoprotein E receptors: normal biology and roles in Alzheimer disease. Cold Spring Harbor Perspect Med. 2012;2(3):a006312.

17. Eisenberg DT, Kuzawa CW, Hayes MG. Worldwide allele frequencies of the human apolipoprotein E gene: climate, local adaptations, and evolutionary history. Am J Phys Anthropol. 2010;143(1):100-11.

18. dbSNP Short Genetic Variations: reference SNP (rs) report. 2021. https:// wwwncbinlmnihgov/snp/rs429358?vertical_tab=true\#frequency_tab. Released April 9, 2021. ; Accessed 4 Jul 2021.

19. Babenko VN, Afonnikov DA, Ignatieva EV, Klimov AV, Gusev FE, Rogaev El. Haplotype analysis of APOE intragenic SNPs. BMC Neurosci. 2018;19(Suppl 1):16-16.

20. González-Castro TB, Tovilla-Zárate CA, Hernández-DíazY, Fresán A, Juárez-Rojop IE, Ble-Castillo JL, López-Narváez L, Genis A, HernándezAlvarado MM. No association between ApoE and schizophrenia: evidence of systematic review and updated meta-analysis. Schizophr Res. 2015;169(1):355-68.

21. Borroni B, Costanzi C, Padovani A. Genetic susceptibility to behavioural and psychological symptoms in Alzheimer disease. Curr Alzheimer Res. 2010;7(2):158-64

22. Panza F, Frisardi V, Seripa D, D'Onofrio G, Santamato A, Masullo C, Logroscino G, Solfrizzi V, Pilotto A. Apolipoprotein E genotypes and neuropsychiatric symptoms and syndromes in late-onset Alzheimer's disease. Ageing Res Rev. 2012;11(1):87-103.

23. Panza F, Seripa D, D'Onofrio G, Frisardi V, Solfrizzi V, Mecocci P, Pilotto A. Neuropsychiatric symptoms, endophenotypes, and syndromes in late-onset Alzheimer's disease: focus on APOE gene. Int J Alzheimer's Dis. 2011;2011:1-14.

24. Egert S, Rimbach G, Huebbe P. ApoE genotype: from geographic distribution to function and responsiveness to dietary factors. Proc Nutr Soc. 2012;71(3):410-24.

25. Radwan ZH, Wang X, Waqar F, Pirim D, Niemsiri V, Hokanson JE, Hamman RF, Bunker CH, Barmada MM, Demirci FY. Comprehensive evaluation of the association of APOE genetic variation with plasma lipoprotein traits in US whites and African blacks. PLoS ONE. 2014;9(12):e114618.

26. Duncan MS, Vasan RS, Xanthakis V. Trajectories of blood lipid concentrations over the adult life course and risk of cardiovascular disease and all-cause mortality: observations from the Framingham Study over 35 years. J Am Heart Assoc. 2019;8(11):e011433.

27. Orozco-Beltran D, Gil-Guillen VF, Redon J, Martin-Moreno JM, PallaresCarratala V, Navarro-Perez J, Valls-Roca F, Sanchis-Domenech C, Fernandez-Gimenez A, Perez-Navarro A. Lipid profile, cardiovascular disease and mortality in a Mediterranean high-risk population: the ESCARVAL-RISK study. PLOS ONE. 2017;12(10):e0186196.

28. Fan Z, Wu Y, Shen J, Ji T, Zhan R. Schizophrenia and the risk of cardiovascular diseases: a meta-analysis of thirteen cohort studies. J Psychiatr Res. 2013;47(11):1549-56.
29. Wu X, Huang Z, Wu R, Zhong Z, Wei Q, Wang H, Diao F, Wang J, Zheng $L$, Zhao J, et al. The comparison of glycometabolism parameters and lipid profiles between drug-naïve, first-episode schizophrenia patients and healthy controls. Schizophr Res. 2013;150(1):157-62.

30. Woods AG, Sokolowska I, Taurines R, Gerlach M, Dudley E, Thome J, Darie CC. Potential biomarkers in psychiatry: focus on the cholesterol system. J Cell Mol Med. 2012;16(6):1184-95.

31. Boiko AS, Mednova IA, Kornetova EG, Semke AV, Bokhan NA, Loonen AJ, Ivanova SA. Apolipoprotein serum levels related to metabolic syndrome in patients with schizophrenia. Heliyon. 2019;5(7):e02033.

32. Demmer LA, Levin MS, Elovson J, Reuben MA, Lusis AJ, Gordon JI. Tissuespecific expression and developmental regulation of the rat apolipoprotein B gene. Proc Natl Acad Sci. 1986;83(21):8102-6.

33. Mayo Clinic Laboratories: APOAB — clinical: apolipoprotein A1 and B, Serum. 2021. https://www.mayocliniclabs.com/test-catalog/Clinical+ and+Interpretive/607593. Accessed 4 Jul 2021.

34. Paré G, Çaku A, McQueen M, Anand SS, Enas E, Clarke R, Boffa MB, Koschinsky M, Wang X, Yusuf S. Lipoprotein (a) levels and the risk of myocardial infarction among 7 ethnic groups. Circulation. 2019;139(12):1472-82.

35. Martins-De-Souza D, Wobrock T, Zerr I, Schmitt A, Gawinecka J, Schneider-Axmann T, Falkai P, Turck CW. Different apolipoprotein E, apolipoprotein $\mathrm{A} 1$ and prostaglandin-H2 D-isomerase levels in cerebrospinal fluid of schizophrenia patients and healthy controls. World J Biol Psychiatry. 2010;11(5):719-28.

36. Wen F, Tan J. Effects of phenothiazine drugs on serum levels of apolipoproteins and lipoproteins in schizophrenic subjects. Acta Pharmacol Sin. 2003;24(10):1001-5.

37. Ozornin A, Govorin N. Blood lipid spectrum changes in first-episode schizophrenia patients treated with risperidone and haloperidol. Eur Psychiatry. 2013;28(S1):1.

38. Mabrouk H, Mechria H, Mechri A, Azizi I, Neffati F, Douki W, Gaha L, Najjar MF. Paraoxonase 1 activity and lipid profile in schizophrenic patients. Asian J Psychiatr. 2014;9:36-40.

39. Walss-Bass C, Lokesh GL, Dyukova E, Gorenstein DG, Roberts DL, Velligan $D$, Volk DE. X-Aptamer technology identifies C4A and ApoB in blood as potential markers for schizophrenia. Mol Neuropsychiatr. 2019;5(1):52-9.

40. Huang JT, Wang L, Prabakaran S, Wengenroth M, Lockstone H, Koethe D, Gerth C, Gross S, Schreiber D, Lilley K. Independent protein-profiling studies show a decrease in apolipoprotein A1 levels in schizophrenia CSF, brain and peripheral tissues. Mol Psychiatry. 2008;13(12):1118-28.

41. Han S, Xu Y, Gao M, Wang Y, Wang J, Liu Y, Wang M, Zhang X. Serum apolipoprotein $E$ concentration and polymorphism influence serum lipid levels in Chinese Shandong Han population. Medicine (Baltimore). 2016;95(50):e5639.

42. Song F, Poljak A, Crawford J, Kochan NA, Wen W, Cameron B, Lux O, Brodaty H, Mather K, Smythe GA. Plasma apolipoprotein levels are associated with cognitive status and decline in a community cohort of older individuals. PLoS ONE. 2012;7(6):e34078.

43. Eichner JE, Dunn ST, Perveen G, Thompson DM, Stewart KE, Stroehla BC. Apolipoprotein E polymorphism and cardiovascular disease: a HuGE review. Am J Epidemiol. 2002;155(6):487-95.

44. Raygani AV, Rahimi Z, Kharazi H, Tavilani H, Pourmotabbed T. Association between apolipoprotein E polymorphism and serum lipid and apolipoprotein levels with Alzheimer's disease. Neurosci Lett. 2006;408(1):68-72.

45. Lumsden AL, Mulugeta A, Zhou A, Hyppönen E. Apolipoprotein E (APOE) genotype-associated disease risks: a phenome-wide, registrybased, case-control study utilising the UK Biobank. EBioMedicine. 2020;59:102954.

46. Williams JR. The declaration of Helsinki and public health. Bull World Health Organ. 2008;86(8):650-2.

47. Kay SR, Fiszbein A, Opler LA. The Positive and Negative Syndrome Scale (PANSS) for Schizophrenia. Schizophr Bull. 1987;13(2):261-76.

48. Si TM, Yang JZ, Shu L, Wang XL, Kong QM, Zhou M, Li XN, Liu C. The reliability, validity of PANSS and its implication (in Chinese). Chin Ment Health J. 2004;18(1):45-7.

49. Tian W, Zeng X-M, Li L-X, Jin H-K, Luo Q-Z, Wang F, Guo S-S, Cao Y. Gender-specific associations between MICA-STR and nasopharyngeal carcinoma in a southern Chinese Han population. Immunogenetics. 2006;58(2):113-21. 
50. Jurinke C, Oeth P, Den Boom DV. MALDI-TOF mass spectrometry: aA versatile tool for high-performance DNA analysis. Mol Biotechnol. 2004;26(2):147-63.

51. An H, Du X, Huang X, Qi L, Jia Q, Yin G, Xiao C, Huang X-F, Ning Y, Cassidy RM. Obesity, altered oxidative stress, and clinical correlates in chronic schizophrenia patients. Transl Psychiatry. 2018;8(1):258.

52. Zhang XY, Tan YL, Cao LY, Wu GY, Xu Q, Shen Y, Zhou DF. Antioxidant enzymes and lipid peroxidation in different forms of schizophrenia treated with typical and atypical antipsychotics. Schizophr Res. 2006;81(2):291-300.

53. Sole X, Guino E, Valls J, Iniesta R, Moreno V. SNPStats: a web tool for the analysis of association studies. Bioinformatics. 2006;22(15):1928-9.

54. Kane JM, Aguglia E, Altamura AC, Gutierrez JLA, Schooler NR. Guidelines for depot antipsychotic treatment in schizophrenia. European neuropsychopharmacology consensus conference in siena, Italy. Eur Neuropsychopharmacol. 1998:8(1):55-66.

55. Lehman A, Lieberman J, Dixon L, McGlashan T, Regier D. Practice guideline for the treatment of patients with schizophrenia, second edition. Am J Psychiatry. 2004;161(2):1-56.

56. Woods SW. Chlorpromazine equivalent doses for the newer atypical antipsychotics. J Clin Psychiatry. 2003;64(6):663-7.

57. Martorell L, Virgos C, Valero J, Coll G, Figuera L, Joven J, Pocoví M, Labad A, Vilella E. Schizophrenic women with the APOE $\varepsilon 4$ allele have a worse prognosis than those without it. Mol Psychiatry. 2001;6(3):307-10.

58. Pickar D, Malhotra AK, Rooney W, Breier A, Goldman D. Apolipoprotein $\mathrm{E} \varepsilon 4$ and clinical phenotype in schizophrenia. Lancet. 1997:350(9082):930-1.

59. Liu W, Breen G, Zhang J, Li S, Gu N, Feng G, Bai S, Shen T, Yu A, Xue H, et al. Association of APOE gene with schizophrenia in Chinese: a possible risk factor in times of malnutrition. Schizophr Res. 2003;62(3):225-30.

60. Schürhoff F, Krebs MO, Szöke A, Loze JY, Goldberger C, Quignon V, Tignol J, Rouillon F, Laplanche JL, Leboyer M. Apolipoprotein E in schizophrenia: a French association study and meta-analysis. Am J Med Genet B Neuropsychiatr Genet. 2003;119(1):18-23.

61. Kecmanović M, Dobričić V, Dimitrijević R, Keckarević D, Savić-Pavićević D, Keckarević-Marković M, Ivkovic M, Romac S. Schizophrenia and apolipoprotein E gene polymorphism in Serbian population. Int J Neurosci. 2010;120(7):502-6.

62. Tovilla-Zarate C, Medellin BC, Fresan A, Apiquian R, Dassori A, Rolando M, Escamilla M, Nicolini H. APOE- $\epsilon 3$ and APOE-219G haplotypes increase the risk for schizophrenia in sibling Pairs. J Neuropsychiatry Clin Neurosci. 2009;21(4):440-4.

63. Jonas K, Clouston S, Li K, Fochtmann LJ, Lencz T, Malhotra AK, Cicero D, Perlman G, Bromet EJ, Kotov R. Apolipoprotein E- $\varepsilon 4$ allele predicts escalation of psychotic symptoms in late adulthood. Schizophr Res. 2019;206:82-8.

64. Al-Asmary SM, Kadasah S, Arfin M, Tariq M, Al-Asmari A. Apolipoprotein E polymorphism is associated with susceptibility to schizophrenia among Saudis. Arch Med Sci. 2015;11(4):869-76.

65. LiW. Association of APOE E2 and low-density lipoprotein with depressive symptoms in Chinese senile schizophrenia inpatients: A cross-sectional study. Schizophrenia Res Cognit. 2021;23:100193.

66. Lieberman JA 3rd. Metabolic changes associated with antipsychotic use. Prim Care Companion J Clin Psychiatry. 2004;6(Suppl 2):8-13.

67. He H, Zhou Y, Yang M, Li X, Xiang Y-T, Luo J. Comparison of olanzapine versus other second-generation antipsychotics in the improvement of insight and medication discontinuation rate in schizophrenia. Shanghai Arch Psychiatry. 2018;30(3):178.

68. Chakrabarti N, Sinha V, Sinha BNP. A study of lipid profile and apolipoproteins $\mathrm{A} 1$ and $\mathrm{B}$ : Their relationship to aggression and psychopathology in male patients with psychosis. J Forensic Psychiatry Psychol. 2004;15(2):314-24.

69. Uludag K, Wang DM, Goodman C, Chen DC, Wang L, Zhang X. Prevalence, clinical correlates and risk factors associated with Tardive Dyskinesia in Chinese patients with schizophrenia. Asian J Psychiatry. 2021;66:102877.

70. Rao W, Zhang Y, Li K, Zhang XY. Association between cognitive impairment and apolipoprotein A1 or apolipoprotein B levels is regulated by apolipoprotein E variant rs429358 in patients with chronic schizophrenia. Aging (Albany NY). 2021;13(12):16353-66.

71. Pillinger T, McCutcheon RA, Vano L, Mizuno Y, Arumuham A, Hindley G, Beck K, Natesan S, Efthimiou O, Cipriani A, et al. Comparative effects of 18 antipsychotics on metabolic function in patients with schizophrenia, predictors of metabolic dysregulation, and association with psychopathology: a systematic review and network meta-analysis. Lancet Psychiatry. 2020;7(1):64-77.

72. Zheng J, Yin Q, Cao J, Zhang B. Obesity contributes more to increasing ApoB/ApoA1 ratio than hyperandrogenism in PCOS women aged 20-38 years in China. Exp Ther Med. 2017;13(4):1337-42.

73. Wang W, Blackett P, Khan S, Lee E. Apolipoproteins A-I, B, and C-III and obesity in young adult cherokee. J Lipids. 2017;2017:8236325.

74. Padmanabhan JL, Tandon N, Haller CS, Mathew IT, Eack SM, Clementz BA, Pearlson GD, Sweeney JA, Tamminga CA, Keshavan MS. Correlations between brain structure and symptom dimensions of psychosis in schizophrenia, schizoaffective, and psychotic bipolar I disorders. Schizophr Bull. 2015;41(1):154-62.

75. Goltermann J, Redlich R, Dohm K, Zaremba D, Repple J, Kaehler C, Grotegerd D, Förster K, Meinert S, Enneking V, et al. Apolipoprotein E homozygous $\varepsilon 4$ allele status: a deteriorating effect on visuospatial working memory and global brain structure. Front Neurol. 2019;10:552.

\section{Publisher's Note}

Springer Nature remains neutral with regard to jurisdictional claims in published maps and institutional affiliations.

Ready to submit your research? Choose BMC and benefit from:

- fast, convenient online submission

- thorough peer review by experienced researchers in your field

- rapid publication on acceptance

- support for research data, including large and complex data types

- gold Open Access which fosters wider collaboration and increased citations

- maximum visibility for your research: over 100M website views per year

At BMC, research is always in progress.

Learn more biomedcentral.com/submissions 